Abstract 069 Table 1

\begin{tabular}{lccc}
\hline Black ethnicity data & $\begin{array}{l}\text { Afro-Caribbean } \\
\text { patients } \mathbf{N = 1 7 0}\end{array}$ & $\begin{array}{l}\text { All other } \\
\text { patients N=972 }\end{array}$ & p Value \\
\hline Age & $71(53-77)$ & $73(63-81)$ & $<0.01$ \\
Male gender & $110(64.7 \%)$ & $652(67.1 \%)$ & $<0.01$ \\
Ischaemic cardiomyopathy & $22(12.9 \%)$ & $406(41.8 \%)$ & $<0.01$ \\
Non-ischaemic (total) & $148(87.1 \%)$ & $566(58.2 \%)$ & $<0.01$ \\
Amyloid (AL and TTR) & $27(15.9 \%)$ & $21(2.2 \%)$ & $<0.01$ \\
ATTR V122I & $\mathbf{1 7}(\mathbf{1 0} \%)$ & $\mathbf{4}(\mathbf{0 . 4 \% )}$ & $<\mathbf{0 . 0 1}$ \\
Dilated cardiomyopathy & $46(27.1 \%)$ & $179(18.4 \%)$ & $<0.01$ \\
Hypertensive heart disease & $32(18.8 \%)$ & $68(7.0 \%)$ & $<0.01$ \\
\hline
\end{tabular}

\section{ENZYME REPLACEMENT THERAPY IMPROVES CARDIAC FEATURES OF FABRY DISEASE}

doi:10.1136/heartjnl-2012-301877b.70

M Motwani, ${ }^{*}$ S Banypersad, P Woolfson, S Waldek. Salford Royal Hospital NHS Foundation Trust, UK

Introduction Fabry disease (FD) is an X linked deficiency of $\alpha$-galactosidase A which leads to an accumulation of globotriaosylceramide (GL-3) throughout the body, particularly in the heart, brain and kidneys. Although left ventricular hypertrophy (LVH) in FD can improve with enzyme-replacement therapy (ERT), the response is difficult to predict. Furthermore, the response of other cardiac features such as aortic dilatation and ECG changes are poorly understood.

Methods A local registry of 66 patients with FD was studied. ECG, echocardiogram and Fabry Outcome Survey-Mainz Severity Score

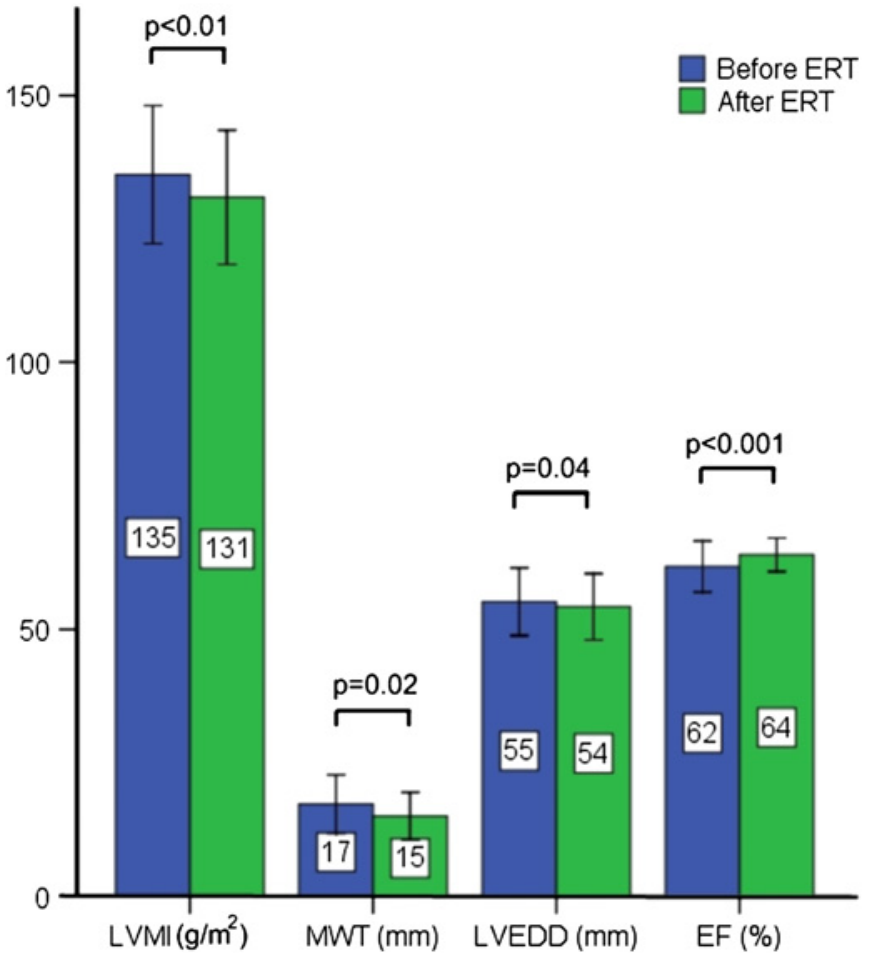

Abstract 070 Figure 1 Effect of enzyme replacement therapy (ERT) on LV morphology and function in patients with LVH at baseline. Left ventricular mass index (LVMI), maximal wall thickness (MWT), LV enddiastolic diameter (LVEDD) and ejection fraction all showed significant improvements (all $p$ values $<0.05$ ) at the end of follow-up (median 3 years). [Values shown are mean \pm SD].
Index (FOS-MSSI) data were compared between baseline and after long-term ERT (median 36 months).

Results In patients with LVH $(n=42)$, left ventricular mass index (LVMI), maximal wall thickness (MWT), left ventricular enddiastolic diameter (LVEDD) and ejection fraction (EF) were all seen to improve after ERT (LVMI: $135 \pm 13$ vs $133 \pm 13 \mathrm{~g} / \mathrm{m}^{2}$, MWT: $17 \pm 6$ vs $16 \pm 5 \mathrm{~mm}$, LVEDD: $55 \pm 6$ vs $54 \pm 6 \mathrm{~mm}$; EF: $62 \pm 5$ vs $64 \pm 3 \%$; $\mathrm{p}<0.05$ ) (Abstract 070 figure 1). In the entire patient group, $P Q$ interval and $\mathrm{P}$ wave duration significantly increased with ERT (PQ: $131 \pm 13$ vs $144 \pm 13 \mathrm{~ms}$, P: $76 \pm 5$ vs $90 \pm 6 \mathrm{~ms}$; p values <0.001); QTc interval significantly decreased $(418 \pm 18$ vs $410 \pm 15 \mathrm{~ms} ; \mathrm{p}<0.001)$; and median FOS-MSSI score fell from 16 to $14(\mathrm{p}<0.001)$ (Abstract 070 figure 2). On logistic-regression analysis, none of the recorded baseline features (age, gender, LVMI, MWT, LVEDD, aortic diameter, EF, PQ interval, P wave duration, ORS duration, OT interval, Romhilt-Estes score or FOS-MSSI) predicted improvements in LVH or FOS-MSSI with ERT ( $p>0.05)$.

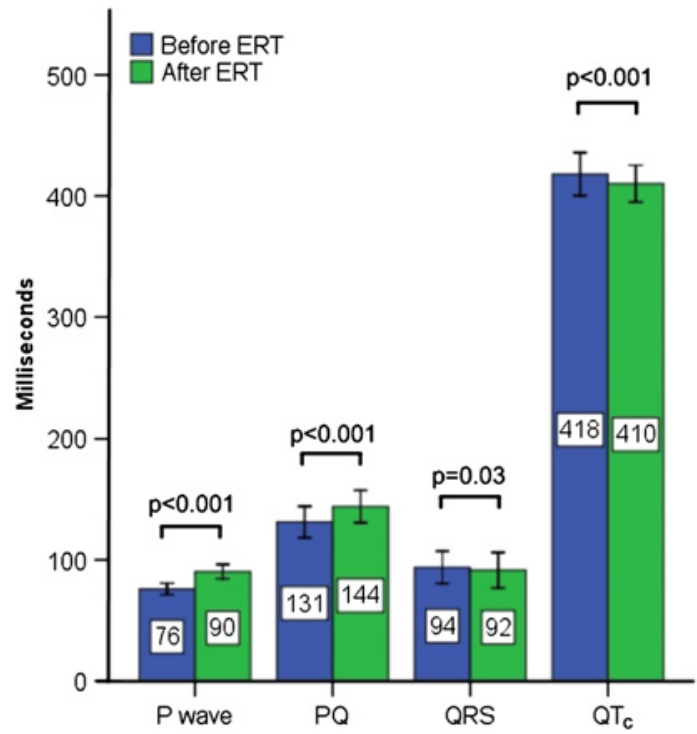

Abstract 070 Figure 2 Effect of enzyme replacement (ERT) on the conduction system. There was a significant increase in $\mathrm{P}$ wave duration and PQ interval from baseline; there was a significant decrease in QRS width and OTc interval from baseline at the end of follow-up (median 3 years). [Values shown are mean \pm SD].

Conclusions ERT improved LV morphology and function in patients with LVH- but there was no relationship between age, gender, FOSMSSI or baseline ECG/TTE features and the response. ERT also normalised long OTc intervals, short PQ intervals and short P waves; and reduced disease burden (FOS-MSSI).

\section{CONCENTRIC HYPERTROPHIC REMODELLING AND SUBENDOCARDIAL DYSFUNCTION IN MITOCHONDRIAL DNA POINT MUTATION CARRIERS}

doi:10.1136/heartjnl-2012-301877b.71

M G D Bates,* K G Hollingsworth, J Newman, D G Jakovljevic, B D Keavney, A M Blamire, G A MacGowan, P F Chinnery, D M Turnbull, R W Taylor, M I Trenell, G S Gorman. Newcastle University, UK

Background Cardiomyopathy is an independent predictor of morbidity and early mortality in mitochondrial DNA disease, and 
occurs in $20 \%-40 \%$ of adult patients harbouring the common m.3243A > G mutation, usually with a hypertrophic phenotype. Pathogenetic mechanisms are unclear yet no detailed study of myocardial structure, function and bioenergetics has been performed in m.3243A $>\mathrm{G}$ mutation carriers to identify early markers of cardiac involvement.

Methods Cardiac MRI was performed in 20 adult patients $(10$ males, mean age $38.7 \pm 13.1$ years) harbouring the m.3243A $>\mathrm{G}$ mutation, without clinical evidence of cardiac involvement on routine ECG and echocardiographic screening, and 20 age- and gender-matched healthy controls (10 males, 38.4 14.2 years): (1) phosphorus-31 magnetic resonance spectroscopy, (2) cine imaging (3), cardiac tagging, and (4) late gadolinium enhancement (LGE) imaging on a Philips Intera Achieva 3 Tesla scanner. Clinical disease burden was determined using: (1) the Newcastle Mitochondrial Disease Adult Scale (NMDAS), a 29-domain validated scoring system with a single cardiac domain, and (2) urinary mutation load, the best predictor of overall clinical outcome in $\mathrm{m} .3243 \mathrm{~A}>\mathrm{G}$ mutation carriers.

Results Compared to control subjects and following Bonferroni correction for multiple comparisons, patients had increased left ventricular mass index (LVMI), LV mass to end-diastolic volume ratio ( $\mathrm{M} / \mathrm{V}$ ratio), LV radial wall thicknesses (all $\mathrm{p}<0.01$ ), peak torsion and torsion to endocardial circumferential strain ratio (both $p<0.05)$. Longitudinal shortening was decreased in patients $(p<0.0001)$ and correlated with increased LVMI $(r=-0.59, p=0.01)$. These findings are consistent with a reduction in contractile function in the subendocardium compared to the subepicardium, and similar to those reported in familial hypertrophic cardiomyopathy, but there were no differences in diastolic function in our study. Nine patients had diabetes mellitus and 3 had treated hypertension. Among patients, there was no correlation of LVMI or $\mathrm{M} / \mathrm{V}$ ratio with age, blood pressures, fasting blood glucose or HbA1C but urinary mutation load and NMDAS score correlated strongly with LVMI ( $r=0.72, p<0.001$ and $r=0.85, p<0.0001$ respectively). Phosphocreatine (PCr)/adenosine triphosphate (ATP) ratio was significantly decreased $(\mathrm{p}=0.003)$ in patients $(1.57 \pm 0.34)$ compared to controls $(1.97 \pm 0.22)$ but there were no associations between $\mathrm{PCr} /$ ATP ratio and markers of myocardial function, or mass. No patients displayed evidence of focal myocardial fibrosis on LGE imaging.

Conclusions Concentric remodelling and subendocardial dysfunction are prevalent in m.3243A $>\mathrm{G}$ mutation carriers without clinical cardiac disease. Assessment of myocardial deformation may be useful in monitoring disease progression or response to early intervention. Patients with higher urinary mutation loads and disease burden may be at increased risk of cardiac involvement.

\section{FRAGMENTED ORS: A PREDICTOR OF MYOCARDIAL SCAR AND FIBROSIS IN HYPERTROPHIC CARDIOMYOPATHY}

doi:10.1136/heartjnl-2012-301877b.72

N Sheikh, ${ }^{*}$ M Papadakis, R Bastiaenen, L Millar, N Emmanuel, S Ghani, A Zaidi, S Gati, N Chandra, E Behr, S Sharma. St. George's University of London, London, UK

Background It is well-established that fragmented ORS complexes (fORS) on the 12-lead ECG are a predictor of delayed gadolinium enhancement (DGE) on Cardiac MRI (CMR) and indicate myocardial scar or fibrosis in patients with coronary artery disease and dilated cardiomyopathy. Moreover, fORS appear to correlate well with arrhythmic events and mortality in these cohorts. However the significance of fORS in hypertrophic cardiomyopathy (HCM) is yet to be established. We sought to determine whether the presence of $\mathrm{fQRS}$ is a predictor of delayed gadolinium enhancement (DGE) on CMR in patients with HCM.

Methods The 12-lead ECGs of 82 consecutive patients with HCM who underwent CMR with gadolinium were analysed for the presence of fORS by two independent readers blinded to the CMR findings. Patients with documented myocardial infarction $(n=3)$ were excluded from further analysis. The ECGs were correlated to CMR findings, and patients separated into DGE positive (DGE+ve; $\mathrm{n}=44$ ) and negative (DGE-ve; $\mathrm{n}=35$ ) groups. ECG territories of fORS were correlated with myocardial segments of DGE on CMR, in order to determine whether areas of fORS predicted areas of DGE. Results Patients from the DGE+ve and DGE-ve groups were of similar gender ( $75 \%$ vs $77 \%$ male respectively, $\mathrm{p}=1.00)$ and age $(54$ $\hat{A} \pm 19$ vs $57 \hat{A} \pm 11$ years respectively, $p=0.41$ ). Fragmented ORS complexes were significantly more prevalent in the DGE+ve group than in the DGE-ve group ( $68.2 \%$ vs $14.3 \%, \mathrm{p}<0.001)$. The positive predictive value (PPV) of fORS for DGE on CMR was $85.7 \%$, with a specificity of $85.7 \%$, sensitivity of $68.2 \%$ and negative predictive value of $68.2 \%$. In the DGE+ve group with fORS ( $n=30)$, fORS ECG lead territory was predictive of regions of DGE on CMRI in $73.3 \%(n=22)$ of patients.

Conclusions The presence of fORS on 12-lead ECG correlates with DGE on CMR in patients with HCM, with good specificity and PPV. Electrocardiographic territories containing fragmentation also correlate with myocardial segments of DGE on CMR. This simple, inexpensive method may therefore be valuable for predicting scar or fibrosis in patients with HCM. Future work should focus on correlating fORS with risk factors and events to determine its use in risk stratification.

\section{EVALUATION OF CLINICAL MARKERS OF EARLY DISEASE EXPRESSION AND THE ABILITY TO PREDICT GENOTYPE IN FAMILIES WITH HCM AND MUTATIONS IN CARDIAC MYOSIN BINDING PROTEIN C}

doi:10.1136/heartjnl-2012-301877b.73

${ }^{1} \mathrm{~S}$ P Page, ${ }^{* 1} \mathrm{~S}$ Kounas, ${ }^{1} \mathrm{P}$ Syrris, ${ }^{2} \mathrm{M}$ I Christiansen, ${ }^{2} \mathrm{~F}$ Rune-Hansen, ${ }^{2} \mathrm{P}$ S Andersen, ${ }^{1} \mathrm{P}$ M Elliott, ${ }^{1} \mathrm{~W}$ J McKenna. ${ }^{1}$ The Heart Hospital, UCLH, UK; ${ }^{2}$ Statens Serum Institut, Denmark

Introduction Familial evaluation for the presence of left ventricular hypertrophy $(\mathrm{LVH})$ is an important part of management in hypertrophic cardiomyopathy. However due to incomplete penetrance, the presence of LVH does not reliably identify all mutation carriers, with consequences for cascade screening. Previous studies in small genotyped populations have suggested that reduced myocardial tissue Doppler velocities in unaffected relatives may predate the development of LVH and may therefore be useful in identifying at risk relatives. The value of such techniques in a large cohort of genotyped relatives remains unknown however. This study sought to prospectively evaluate ECG and Echo markers of early disease expression in a large genotyped cohort of families with mutations in myosin binding protein C (MYBPC3).

Methods Relatives of index cases with HCM related to mutations in MYBPC3 (4 insertion/deletion, 7 missense, 4 nonsense, 5 intronic, 2 double heterozygotes) were evaluated. Clinical examination, ECG and transthoracic Echo (operator blinded to genetic status) were performed and combined with genetic predictive testing. The clinical value of ECG and Echo derived indices in predicting genotype were assessed.

Results Of 95 relatives, 40 did not carry the family mutation (Group 1, 22 males, $38.5 \pm 16.7$ years), 39 were unaffected mutation carriers (Group 2, 17 males, 37.4 \pm 16.8 years) and 16 were clinically affected and excluded from the study. ECG evidence of left atrial enlargement ( 21 vs $3 \%, p=0.01$ ) and non-pathological $Q$ waves ( 64 vs $28 \%$, $\mathrm{p}=0.001$ ) were more common in group 2 than group 1 . S wave amplitude in lead V2 was greater in group 2 than group 1 (16.6 \pm 7.9 vs $12.4 \pm 8.2 \mathrm{mV}, \mathrm{p}=0.02)$ and also lead $\mathrm{V} 3(12.7 \pm 6.9$ vs $9.3 \pm 6.1 \mathrm{mV}$, $\mathrm{p}=0.02)$. ECG criteria for left ventricular hypertrophy were specific 